\title{
Estimativa da produção agropecuária de assentamentos rurais de Mato Grosso do
}

\section{Sul utilizando lógica fuzzy}

Estimated agriculltural and livestock production in rural settlements in Mato Grosso do Sull using

fuzzy logic

Estimación de la producción agrícola en asentamientos rurales en Mato Grosso do Sul mediante lógica difusa

Raul Asseff Castelao

ORCID: https://orcid.org/0000-0002-4677-6088 Universidade Anhanguera, Brasil E-mail: raulassefcastelao@gmail.com

Wildson Antonio de Oliveira Bezerra ORCID: https://orcid.org/0000-0001-7560-8836

Universidade Anhanguera, Brasil

E-mail: wildsonbezerra@gmail.com

Adriane Cristina Correa Soares Veronez ORCID: https://orcid.org/0000-0003-1631-6336

Universidade Anhanguera, Brasil

E-mail: adriane.veronez@gmail.com

Gilberto de Souza Bruno

ORCID: https://orcid.org/0000-0003-3356-2184

Universidade Anhanguera, Brasil

E-mail: gilberto.bruno@gmail.com

Celso Correia de Souza

ORCID: https://orcid.org/0000-0002-2689-8264

Universidade Anhanguera, Brasil E-mail: csouza939@gmail.com

José Antonio Maior Bono

ORCID: https://orcid.org/0000-0002-9573-9494 Universidade Anhanguera, Brasil E-mail: bono@uniderp.edu

Larissa de Fátima Cardoso Duarte ORCID: https://orcid.org/0000-0003-4375-6708

Universidade Anhanguera, Brasil

E-mail: larissa_hermeto@yahoo.com.br

\begin{abstract}
Resumo
É muito importante conhecer as produtividades dos principais produtos da lavoura e da pecuária dos assentamentos de uma região, para que sejam facilitadas as políticas públicas visando a melhoria de vida dos seus assentados. O objetivo deste estudo foi estimar a produtividade da agricultura de da pecuária dos assentamentos rurais de Mato Grosso do Sul (MS). Desse modo foram estimadas as produtividades da agricultura e da pecuária relativas ao milho, feijão e mandioca, e bovina, suína e aves, respectivamente. A amostra constou de vinte e seis assentamentos rurais de MS, sendo utilizados os dados socioeconômicos e demográficos dos assentados de cada assentamento que produziam os cultivares e os animais utilizados na pesquisa. As variáveis estudadas foram, relativos a agricultura, as produções de milho, feijão e mandioca e, relativas à pecuária, a bovina, suína e aves. Com as médias dessas produtividades e, de posse dos níveis de produtividades de cada variável em MS fornecidas por especialistas nos assuntos, aplicou-se a lógica fuzzy para facilitar as decisões sobre a produtividade na agricultura e na pecuária de assentamentos rurais de MS, sobre aqueles dados que se situavam entre o completamente verdadeiro e o completamente falso. Pelos resultados obtidos foram constatadas produtividades médias tanto no setor agrícola quanto no da pecuária dos assentamentos rurais de MS.
\end{abstract}

Palavras-chave: Produtividade da agricultura; Produtividade da pecuária; Níveis de produtividade; Decisão com fuzzy. 


\begin{abstract}
It is very important to know the productivity of the main products of farming and livestock in the settlements of a region so that public policies are aimed at improving the life of its settlers. The objective of this study was to estimate the productivity of agriculture and livestock in rural settlements in Mato Grosso do Sul (MS).In this way, the productivity of agriculture and livestock related to corn, beans and manioc, and bovine, swine and poultry, respectively, were estimated. The sample consisted of twenty-six rural settlements in MS, using the socioeconomic and demographic data of the settlers in each settlement that produced the cultivars and animals used in the research. The variables studied were related to agriculture, the production of corn, beans and cassava and, related to livestock, beef, pork and poultry. With the averages of these productivities and, in possession of the levels of productivity of each variable in MS provided by specialists in the subjects, the fuzzy logic was applied to facilitate decisions about productivity in agriculture and livestock in rural settlements in MS, about those data that were between the completely true and the completely false. The results obtained showed average productivity both in the agricultural and livestock sectors of rural settlements in MS.
\end{abstract}

Keywords: Agriculture productivity; Livestock productivity; Productivity levels; Fuzzy decision.

\title{
Resumen
}

Es muy importante conocer la productividad de los principales productos de la agricultura y la ganadería en los asentamientos de una región, para que las políticas públicas estén orientadas a mejorar la vida de sus pobladores. El objetivo de este estudio fue estimar la productividad de la agricultura y la ganadería en asentamientos rurales en Mato Grosso do Sul (MS). De esta forma se estimó la productividad de la agricultura y ganadería relacionada con maíz, frijol y mandioca, y bovina, porcina y avícola, respectivamente. La muestra consistió en veintiséis asentamientos rurales en MS, utilizando los datos socioeconómicos y demográficos de los pobladores de cada asentamiento que produjeron los cultivares y animales utilizados en la investigación. Las variables estudiadas estuvieron relacionadas con la agricultura, la producción de maíz, frijol y yuca y, relacionadas con la ganadería, la carne de vacuno, porcina y avícola. Con los promedios de estas productividades y, en posesión de los niveles de productividad de cada variable en MS proporcionados por especialistas en los temas, se aplicó la lógica difusa para facilitar decisiones sobre productividad en la agricultura y ganadería en asentamientos rurales de MS, sobre aquellos datos que estaban entre completamente verdadero y completamente falso. Los resultados obtenidos mostraron una productividad media tanto en el sector agrícola como ganadero de los asentamientos rurales de la EM.

Palabras clave: Productividad agrícola; Productividad ganadera; Niveles de productividad; Decisión borrosa.

\section{Introdução}

O conceito de produtividade de um produtor rural está ligado ao seu sistema de produção, que é um conjunto de elementos inter-relacionados que mede a relação entre o que foi produzido e os insumos utilizados para tal, num certo intervalo de tempo. É muito importante a análise de produtividade de assentamentos rurais de Mato Grosso do Sul (MS) para as tomadas de decisões das autoridades competentes, principalmente, do Instituto Nacional de Colonização e Reforma Agrária (INCRA), do Serviço Nacional de Aprendizagem Rural (SENAR) e da Agência de Desenvolvimento Agrário e Extensão Rural (AGRAER), responsáveis pelos assentamentos de MS (Oliveira et al., 2017).

Existem dificuldades na determinação dessas produtividades, pois, a maioria dos assentamentos cultivam vários cereais e criam diversos tipos de animais, o que torna difícil a determinação de suas produtividades devido às incertezas advindas nas decisões envolvendo diferentes fatores de produção. A lógica fuzzy pode auxiliar nas tomadas de decisão sobre a produtividade, pois, permite o desenvolvimento de sistemas que representam decisões humanas fazendo uso de inferências, proporcionando resultados mais confiáveis se comparados com soluções utilizando a decisão humana.

O objetivo deste artigo foi estimar a produtividade da agropecuária dos assentamentos rurais utilizando lógica fuzzy, tomando como base os fatores importantes de produção para desses assentamentos, de modo a torná-los unidades de produção estruturadas inseridas de forma competitiva no processo de produção agropecuária regional. Para a consecução desse objetivo, esse foi dividido em três objetivos específicos: determinar os níveis de produtividades de assentamentos rurais de MS; utilizar a lógica fuzzy para modelar as incertezas presentes nos cálculos de produtividade. 
Considerando um cenário regional, pode-se aplicar essa sistemática ao estado de Mato Grosso do Sul (MS), cujo desenvolvimento econômico tem destaque para vocação na agropecuária, grande setor do MS que corresponde a 18\% de seu Produto Interno Bruto (PIB) e de 5\% de participação do setor no PIB do Brasil (IBGE, 2015).

Mato Grosso do Sul é um dos 27 Estados da federação brasileira e possui, aproximadamente, 36 milhões de hectares, com população estimada em 2,7 milhões de habitantes e 79 municípios em sua divisão territorial (IBGE, 2020). A qualidade do clima e água existente no Estado são propicias às diversas atividades do agronegócio tendo predominância de pastagens nativas (Bungenstab, 2012).

Ao longo destes 20 anos, o estado de MS passou de 72 municípios em 1991 para 77 em 2000 e 78 em 2010 e, com a criação de Paraíso das Águas em 2013, tem atualmente 79 municípios. Conforme o caderno estatístico da Secretaria de Estado de Meio Ambiente e Desenvolvimento Econômico (SEMADE), a população de MS apresenta um crescimento médio de 17\% levando em consideração 1991 como ano base. A taxa de urbanização passou de 79,45\% em 1991 para 84,08\% em 2000 e 85,64\% em 2010 (Castelao et al., 2020).

Lima (2014) coloca que no ranking entre as unidades da Federação, Mato Grosso do Sul ocupa a $17^{\mathrm{a}}$ posição no PIB nacional e a $10^{\mathrm{a}}$ posição no PIB per capita. A economia está baseada no setor primário e terciário, mas vem tendo ótimos desempenhos no setor industrial nos últimos anos. Para subsidiar o processo da pesquisa foram utilizados os métodos de investigação histórico e comparativo. A partir deste estudo, buscou-se identificar a existência de crescimento econômico e social concomitante com a preservação e manutenção do meio ambiente das localidades estudadas.

Fagundes et al. (2016) descreve que o Estado tem se destacado no cenário nacional e também internacional em função as suas características propicias a produção agroindustrial e pela oportunidade na possibilidade de pioneirismo no desenvolvimento de novas tecnologias como, por exemplo, biodiesel.

O estado encontra-se em uma região considerada estratégica na ótica da biodiversidade uma vez que lhe permite possuir dentro de seu território, contato com diversos macro sistemas, podendo transformar esta rica biodiversidade em potencial econômico, social e cultura e, além disso, possui também a característica intrínseca na contribuição da manutenção da biodiversidade brasileira (Graciolli et al., 2017).

Coutinho et al. (2019) aponta que as principais atividades na criação de empregos foram do setor de serviços (administração pública, serviços e comércio), no entanto, as atividades de indústria e ligadas ao agronegócio passam a ter destaque nos últimos anos sendo, portanto, importante indutores de emprego e renda na economia regional.

\section{Metodologia}

A pesquisa é de natureza quantitativa e procura abordar, a partir da estrutura quantitativa, os principais resultados dos artigos selecionados para ampliar a discussão do trabalho.

O objeto desta pesquisa foi o conjunto de 30 assentamentos rurais do estado de MS. Foram utilizados dados secundários quantitativos que envolviam aspectos demográficos, socioeconômicos e ambientais, oriundos de um banco de dados do INCRA (MS) sobre assentamentos de MS, no ano de 2015. As variáveis quantitativas englobaram dados sobre a produção de feijão, milho e mandioca, o número de bovinos, suínos, aves, em cada assentamento, bem como, as respectivas áreas de produção, de forma à estimar o nível de produtividade em cada assentamento rural.

O índice final de produtividade de cada variável foi obtido pela média aritmética das produtividades da variável em todos os assentamentos amostrados. A produtividade que se busca analisar está baseada em duas atividades dos assentamentos: a pecuária e a agricultura. Na agricultura: as produtividades de feijão, milho e mandioca foram dadas na unidade toneladas por hectare (t ha-1). Na pecuária, as produtividades bovina, suína e de aves foram dadas pelas unidades de animais por hectare (ua ha-1). 
De posse das produtividades dos assentamentos, bem como da média das produtividades para o conjunto de assentamentos para cada produto e, considerando os níveis de produtividade para as seis variáveis estabelecidas por especialistas da área, pode-se aplicar conceitos de lógica fuzzy no conjunto de produtividades resultantes, que foi aproximado por um número difuso, de função de pertinência triangular que representou o desempenho do assentamento segundo a ótica pecuária ou agricultura.

De acordo com o órgão Produção Agrícola Municipal (PAM), do IBGE (2015) e CONAB (2007), a média de produtividade do estado de MS no setor agrícola relacionado à produção de feijão, milho e mandioca, calculado em kg ha-1 está no Quadro 1.

Quadro 1. Níveis de produtividade da agricultura em (t ha-1) e na pecuária (ua há-1), com níveis: abaixo do Ideal, Ideal e Acima do Ideal em Mato Grosso do Sul, em 2015.

\begin{tabular}{|l|l|l|l|l|}
\hline \multirow{2}{*}{ Cultivar } & \multicolumn{3}{l|}{ Níveis de Produtividade } \\
\cline { 3 - 5 } \multicolumn{2}{|c|}{} & Abaixo do Ideal & Ideal & Acima do Ideal \\
\hline \multirow{3}{*}{ Agrícola } & Milho $\left(\mathrm{t} \mathrm{ha}^{-1}\right)$ & $<6$ & 6 a 8 & $>8$ \\
\cline { 2 - 5 } & Feijão $\left(\mathrm{t} \mathrm{ha}^{-1}\right)$ & $<1,5$ & 1,5 a 2 & $>2$ \\
\cline { 2 - 5 } & Mandioca $\left(\mathrm{t} \mathrm{ha}^{-1}\right)^{*}$ & $<15$ & 15 a 20 & $>20$ \\
\hline \multirow{3}{*}{ Pecuária } & Aves $\left(\text { ua ha }^{-1}\right)^{*}$ & $<20$ & 20 a 50 & $>50$ \\
\cline { 2 - 5 } & Bovino $\left(\right.$ ua ha $\left.^{-1}\right)$ & $<1$ & 1 a 2 & $>2$ \\
\cline { 2 - 6 } & Suíno $\left(\right.$ ua ha $\left.^{-1}\right)$ & $<7$ & 7 a 10 & $>10$ \\
\hline
\end{tabular}

Fonte: Autores.

Como resultado das inferências foi necessário determinar uma variável fuzzy, que foi "estado (saída) e escolher os valores fuzzy de ação (fuzzificação), que foram três valores, "Abaixo do Ideal", "Ideal” e "Acima do Ideal”, cujos valores estão no Quadro 2.

Quadro 2. Pertinências de saídas da inferência sobre a produtividade agrícola e pecuária dos assentamentos de MS, em 2015

\begin{tabular}{|l|l|}
\hline Estado & Níveis de Produtividade \\
\hline Abaixo do Ideal & $<-5$ \\
\hline Ideal & $-5 \mathrm{a}+5$ \\
\hline Acima do Ideal & $>5$ \\
\hline
\end{tabular}

Fonte: Autores.

As inferências utilizadas foram adaptadas de Ramos et al. (2015), correspondentes aos cultivares considerados no estudo: feijão, milho e mandioca e, como saída está contemplada a produtividade (Quadro 3). 
Quadro 3. Inferências de entradas correspondentes à agricultura e à pecuária e, como saída, o nível de produtividade

\begin{tabular}{|c|c|c|c|c|}
\hline & $\begin{array}{l}\text { SE Produtividade do } \\
\text { feijão ou bovina é: }\end{array}$ & $\begin{array}{l}\text { e SE } \\
\text { Produtividade do milho ou } \\
\text { suína é: }\end{array}$ & $\begin{array}{l}\text { e SE } \\
\text { Produtividade da mandioca ou } \\
\text { aves é: }\end{array}$ & $\begin{array}{l}\text { ENTÃO } \\
\text { Produtividade do assentamento é: }\end{array}$ \\
\hline 1 & Baixa & Baixa & Baixa & Baixa \\
\hline 2 & Baixa & Baixa & Média & Baixa \\
\hline 3 & Baixa & Média & Baixa & Baixa \\
\hline 4 & Média & Baixa & Baixa & Baixa \\
\hline 5 & Baixa & Média & Média & Média \\
\hline 6 & Média & Baixa & Média & Média \\
\hline 7 & Média & Média & Baixa & Média \\
\hline 8 & Média & Média & Média & Média \\
\hline 9 & Alta & Baixa & Baixa & Média \\
\hline 10 & Baixa & Alta & Baixa & Média \\
\hline 11 & Baixa & Baixa & Alta & Média \\
\hline 12 & Baixa & Alta & Alta & Média \\
\hline 13 & Alta & Baixa & Alta & Média \\
\hline 14 & Alta & Alta & Baixa & Média \\
\hline 15 & Alta & Alta & Média & Alta \\
\hline 16 & Alta & Média & Alta & Alta \\
\hline 17 & Média & Alta & Alta & Alta \\
\hline 18 & Alta & Média & Média & Média \\
\hline 19 & Média & Média & Alta & Média \\
\hline 20 & Alta & Alta & Alta & Alta \\
\hline
\end{tabular}

Fonte: *Adaptado de Ramos et al. (2015).

A inferência é uma etapa que serve de suporte para a tomada de decisão, pois, nela são determinados os graus de pertinência de cada elemento ao conjunto para posterior utilização das regras do tipo "Se - Então". Ao contrário da "fuzzificação", que transforma um dado quantitativo em um termo nebuloso, a "defuzzificação" transforma o dado nebuloso em dado quantitativo.

\section{Resultados e Discussão}

Observou-se, que os dados de alguns assentamentos apresentavam incoerências, ou não produziram algum dos produtos em análises foram eliminados do processo. O Quadro 4 apresenta as áreas de produção e as quantidades médias produzidas dos cereais feijão, milho e mandioca nos assentamentos de MS, em 2015. 
Quadro 4. Áreas de produção e as quantidades médias produzidas dos cereais feijão, milho e mandioca, selecionados para a pesquisa, nos assentamentos de MS, em 2015.

\begin{tabular}{|c|c|c|c|c|c|c|}
\hline \multirow[b]{2}{*}{ Assentamento } & \multicolumn{2}{|l|}{ Feijão } & \multicolumn{2}{|l|}{ Milho } & \multicolumn{2}{|c|}{ Mandioca } \\
\hline & $\begin{array}{l}\text { Área } \\
\text { Média }\end{array}$ & $\begin{array}{l}\text { Volume } \\
\text { Médio }\end{array}$ & $\begin{array}{l}\text { Área } \\
\text { Média }\end{array}$ & $\begin{array}{l}\text { Volume } \\
\text { Médio }\end{array}$ & $\begin{array}{l}\text { Área } \\
\text { Média }\end{array}$ & $\begin{array}{l}\text { Volume } \\
\text { Médio }\end{array}$ \\
\hline Alambari & 0,18 & 247,50 & 0,35 & 230,00 & 0,30 & 1278,00 \\
\hline Altemir Tortelli & - & - & 1,13 & 325,00 & 0,38 & 1200,00 \\
\hline Corguinho & 0,60 & 630,00 & - & - & 0,28 & 305,00 \\
\hline Liberdade Camponesa & 0,65 & 810,00 & - & - & 0,73 & $2.337,50$ \\
\hline Torre de Pedra & - & - & - & - & - & - \\
\hline Rancho Alegre & 0,10 & - & - & 0,03 & 0,10 & 625,00 \\
\hline Santa Mônica & 0,25 & 420,00 & 0,88 & $10.075,00$ & 0,50 & 325,00 \\
\hline Estrela Campo Grande & 0,13 & 270,00 & 0,94 & $1.087,50$ & 0,75 & $7.125,00$ \\
\hline Estrela Jaraguari & 0,05 & - & - & - & 0,05 & - \\
\hline Indaiá & - & - & 0,08 & - & 0,20 & 137,50 \\
\hline Santo Antônio & - & - & 0,13 & - & 0,13 & $2.500,00$ \\
\hline Piuva & 0,68 & 900,00 & - & - & - & 0,75 \\
\hline Sete de Setembro & 0,50 & - & 0,05 & - & 0,20 & - \\
\hline Barra Nova & - & - & - & - & 1,63 & 437,50 \\
\hline Lagoa Azul & - & - & 2,63 & $16.560,00$ & 1,53 & 153,25 \\
\hline Nazareth & 0,30 & 720,00 & 0,25 & 20,00 & 0,18 & 125,50 \\
\hline Ranildo da Silva & 0,25 & 330,00 & 0,50 & 4,50 & 2,25 & 326,25 \\
\hline Eldorado (441) & 0,13 & 315,00 & 0,40 & 3,00 & 0,35 & 375,00 \\
\hline Tejim & - & - & - & - & - & - \\
\hline Eldorado II & 0,13 & - & 0,13 & $3.745,00$ & 1,75 & 375,00 \\
\hline Angélica & - & - & - & - & 0,25 & $1.250,00$ \\
\hline Volta Redonda Cut & - & - & - & - & - & - \\
\hline Volta Redonda Fat & 0,08 & - & - & - & 0,08 & - \\
\hline Santa Luzia & 0,13 & - & 0,43 & 2,50 & 0,05 & - \\
\hline São João & 0,13 & - & - & - & - & - \\
\hline Sucesso & 0,63 & 1080,00 & 0,80 & 250,00 & 0,88 & 532,50 \\
\hline
\end{tabular}

Fonte: Autores.

O Quadro 5 apresenta as áreas de produção e as quantidades médias produzidas e áreas de produções de bovinos, suínos e aves, no ano de 2015. 
Quadro 5. Quantidades médias das áreas de produções, de bovinos, suínos e aves selecionados para a pesquisa nos assentamentos de MS, em 2015

\begin{tabular}{|c|c|c|c|c|c|c|}
\hline \multirow[b]{2}{*}{ Assentamento } & \multicolumn{2}{|c|}{ Bovinos } & \multicolumn{2}{|l|}{ Suinos } & \multicolumn{2}{|l|}{ Aves } \\
\hline & $\begin{array}{l}\text { Área } \\
\text { Média }\end{array}$ & $\begin{array}{l}\text { Qde. } \\
\text { Média }\end{array}$ & $\begin{array}{l}\text { Área } \\
\text { Média }\end{array}$ & $\begin{array}{l}\text { Qde. } \\
\text { Média }\end{array}$ & $\begin{array}{l}\text { Área } \\
\text { Média }\end{array}$ & $\begin{array}{l}\text { Qde. } \\
\text { Média }\end{array}$ \\
\hline Alambari & 5,50 & 16,50 & 0,95 & 13,75 & 0,85 & 68,75 \\
\hline Altemir Tortelli & 3,55 & 36,00 & 1,38 & 10,25 & 1,98 & 192,00 \\
\hline Corguinho & 5,75 & 1,00 & 0,50 & 0,50 & 6,88 & 19,25 \\
\hline Liberdade Camponesa & 3,50 & 0,00 & 0,25 & 0,50 & 3,10 & 10,75 \\
\hline Torre de Pedra & 6,25 & 14,25 & 0,75 & 5,25 & 3,25 & 13,25 \\
\hline Rancho Alegre & 7,50 & 7,50 & 0,13 & 0,75 & 3,13 & 20,00 \\
\hline Santa Mônica & 3,00 & 5,50 & 0,00 & 0,00 & 1,75 & 7,50 \\
\hline Estrela Campo Grande & 0,25 & 0,50 & 2,49 & 5,00 & 2,05 & 28,00 \\
\hline Estrela Jaraguari & 4,25 & 6,50 & 0,03 & 3,00 & 2,78 & 75,00 \\
\hline Indaiá & 1,60 & 2,50 & 0,00 & 1,25 & 1,84 & 15,00 \\
\hline Santo Antônio & 5,50 & 7,75 & 0,00 & 0,00 & 0,18 & 8,50 \\
\hline Piuva & 6,00 & 11,00 & 0,38 & 1,25 & 0,55 & 33,75 \\
\hline Sete de Setembro & 8,78 & 10,00 & 0,13 & 1,00 & 3,13 & 9,00 \\
\hline Barra Nova & 7,25 & 14,75 & 0,35 & 4,75 & 0,91 & 52,50 \\
\hline Lagoa Azul & 3,20 & 18,25 & 0,10 & 1,75 & 0,50 & 42,50 \\
\hline Nazareth & 2,00 & 0,00 & 0,75 & 3,50 & 6,14 & 29,25 \\
\hline Ranildo da Silva & 7,43 & 11,25 & 0,25 & 2,00 & 0,75 & 36,25 \\
\hline Eldorado (441) & 4,75 & 6,50 & 0,38 & 1,50 & 2,63 & 57,50 \\
\hline Tejim & 15,58 & 30,50 & 0,24 & 0,25 & 1,75 & 21,25 \\
\hline Eldorado II & 5,25 & 8,75 & 0,30 & 1,50 & 0,70 & 23,00 \\
\hline Angélica & 8,50 & 22,25 & 0,55 & 2,00 & 1,38 & 20,50 \\
\hline Volta Redonda Cut & 2,75 & 4,50 & 0,50 & 1,00 & 3,45 & 58,25 \\
\hline Volta Redonda Fat & 5,38 & 17,75 & 0,50 & 5,25 & 1,90 & 18,75 \\
\hline Santa Luzia & 5,25 & 6,75 & 0,25 & 3,00 & 3,43 & 35,75 \\
\hline São João & 5,00 & 11,50 & 0,23 & 0,25 & 1,13 & 34,00 \\
\hline Sucesso & 2,93 & 6,75 & 0,03 & 3,00 & 0,55 & 42,25 \\
\hline
\end{tabular}

Fonte: Autores.

Considerando a área média de produção e a produção média de feijão nos dez assentamentos que tinham produção desse cereal, calculou-se a produtividade, em t ha-1, cujos valores estão na Tabela 1. 
Tabela 1. Áreas de produção, volume produzido, produtividades e produtividade média de feijão nos assentamentos selecionados de MS, em 2015.

\begin{tabular}{lllll}
\hline Assentamento & $\begin{array}{l}\text { Área } \\
\text { (ha) }\end{array}$ & $\begin{array}{l}\text { Produção } \\
(\mathbf{k g})\end{array}$ & $\begin{array}{l}\text { Produtividade } \\
\left(\mathbf{k g ~ h a}^{-\mathbf{1}}\right)\end{array}$ & $\begin{array}{l}\text { Produtividade } \\
\left(\mathbf{t ~ h a} \mathbf{~}^{\mathbf{- 1}}\right)\end{array}$ \\
\hline Alambari & 0,18 & 247,50 & 1375,00 & 1,375 \\
Corguinho & 0,60 & 630,00 & 1050,00 & 1,050 \\
Liberdade Camponesa & 0,65 & 810,00 & 1246,15 & 1,246 \\
Santa Mônica & 0,25 & 420,00 & 1680,00 & 1,680 \\
Estrela Campo Grande & 0,13 & 270,00 & 2076,92 & 2,077 \\
Piuva & 0,68 & 900,00 & 1323,53 & 1,324 \\
Nazareth & 0,30 & 720,00 & 2400,00 & 2,400 \\
Ranildo da Silva & 0,25 & 330,00 & 1320,00 & 1,320 \\
Eldorado (441) & 0,13 & 315,00 & 2423,08 & 2,423 \\
Sucesso & 0,63 & 1080,00 & 1714,29 & 1,714 \\
\hline & \multicolumn{5}{c}{ Produtividade Geral Média } \\
\hline
\end{tabular}

Fonte: Autores.

$\mathrm{Na}$ Tabela 2 estão elencadas as áreas de produção em hectares, a produção em quilogramas e as produtividades, em t ha-1, da produção de milho nos 11 assentamentos de MS que produziram esse cereal em 2015.

Tabela 2. Áreas de produção, volume produzido, produtividades e produtividade média de milho nos assentamentos selecionados de MS, em 2015.

\begin{tabular}{lllll}
\hline Assentamento & $\begin{array}{l}\text { Área } \\
(\mathbf{h a})\end{array}$ & $\begin{array}{l}\text { Produção } \\
(\mathbf{k g})\end{array}$ & $\begin{array}{l}\text { Produtividade Média (kg } \\
\left.\mathbf{h a}^{-1}\right)\end{array}$ & $\begin{array}{l}\text { Produtividade Média (t ha' } \\
\mathbf{1})\end{array}$ \\
\hline Alambari & 0,35 & 2250 & 6428,57 & 6,429 \\
Altemir Tortelli & 1,13 & 8125 & 7190,27 & 7,190 \\
Santa Mônica & 0,88 & 6750 & 7670,45 & 7,670 \\
Estrela Campo Grande & 0,94 & 6500 & 6914,89 & 6,915 \\
Lagoa Azul & 2,63 & 16000 & 6083,65 & 6,084 \\
Nazareth & 0,25 & 1250 & 5000,00 & 5,000 \\
Ranildo da Silva & 0,50 & 2000 & 4000,00 & 4,000 \\
Eldorado (441) & 0,40 & 2125 & 5312,50 & 5,313 \\
Eldorado II & 0,13 & 750 & 5769,23 & 5,769 \\
Santa Luzia & 0,43 & 1925 & 4476,74 & 4,477 \\
Sucesso & 0,80 & 3500 & 4375,00 & 4,375 \\
\hline
\end{tabular}

Produtividade Geral Média $\quad \mathbf{5 , 7 4 7}$

Fonte: Autores.

Na Tabela 3 estão anotadas as áreas de produção em hectares, as produções em quilogramas e as produtividades em t ha-1, das produções dos 17 assentamentos de MS que cultivavam mandioca em 2015. 
Tabela 3. Áreas de produção, volume produzido, produtividades e produtividade geral média de mandioca nos assentamentos selecionados de MS, em 2015.

\begin{tabular}{llllc}
\hline Assentamento & $\begin{array}{l}\text { Área } \\
\text { (ha) }\end{array}$ & $\begin{array}{l}\text { Produção } \\
(\mathbf{k g})\end{array}$ & $\begin{array}{l}\text { Produtividade } \\
\left(\mathbf{k g ~ h a}^{-\mathbf{1}}\right)\end{array}$ & $\begin{array}{c}\text { Média } \\
\text { Produtividade Média }\left(\mathbf{t} \mathbf{~ h a}^{-\mathbf{1}} \mathbf{)}\right.\end{array}$ \\
\hline Alambari & 0,3000 & 4300,00 & 14333,33 & 14,333 \\
Altemir Tortelli & 0,3750 & 3500,00 & 9333,33 & 9,333 \\
Corguinho & 0,2750 & 4800,00 & 17454,55 & 17,455 \\
Liberdade Camponesa & 0,7250 & 6700,00 & 9241,38 & 9,241 \\
Rancho Alegre & 0,1000 & 625,00 & 6250,00 & 6,250 \\
Santa Mônica & 0,5000 & 8700,00 & 17400,00 & 17,400 \\
Estrela Campo Grande & 0,7500 & 7125,00 & 9500,00 & 9,500 \\
Indaiá & 0,2000 & 3680,00 & 18400,00 & 18,400 \\
Santo Antônio & 0,1250 & 2500,00 & 20000,00 & 20,000 \\
Barra Nova & 1,6250 & 18700,00 & 11507,69 & 11,508 \\
Lagoa Azul & 1,5250 & 16980,00 & 11134,43 & 11,134 \\
Nazareth & 0,1750 & 3698,00 & 21131,43 & 21,131 \\
Ranildo da Silva & 2,2500 & 23450,00 & 10422,22 & 10,422 \\
Eldorado (441) & 0,3500 & 8760,00 & 25028,57 & 25,029 \\
Eldorado II & 1,7500 & 19890,00 & 11365,71 & 11,366 \\
Angélica & 0,2500 & 4030,00 & 16120,00 & 16,120 \\
Sucesso & 0,8750 & 9809,00 & 11210,29 & 11,210 \\
\hline
\end{tabular}

Fonte: Autores.

Na Tabela 4 estão anotadas as áreas de produção em hectares, os números de cabeças de bovinos (em ua) e as produtividades, em ua ha-1, das produções dos 24 assentamentos de MS que tinham criações de bovinos em 2015. 
Tabela 4. Áreas médias de produção, quantidade média de cabeças de bovinos (UA), produtividades e produtividade geral média de bovinos nos assentamentos selecionados de MS, em 2015.

\begin{tabular}{llll}
\hline Assentamento & $\begin{array}{l}\text { Área } \\
\text { (ha) }\end{array}$ & $\begin{array}{l}\text { Quantidade } \\
\text { Média (ua) }\end{array}$ & $\begin{array}{l}\text { Produtividade } \\
\text { Média }\left(\mathbf{u a ~ h a ~}^{-1}\right)\end{array}$ \\
\hline Alambari & 5,5 & 16,5 & 1,52 \\
Altemir Tortelli & 3,55 & 36,00 & 5,14 \\
Corguinho & 5,75 & 1,00 & 0,09 \\
Torre de Pedra & 6,25 & 14,25 & 1,16 \\
Rancho Alegre & 7,5 & 7,5 & 0,51 \\
Santa Mônica & 3,00 & 5,5 & 0,93 \\
Estrela $\quad$ Campo & 0,25 & 0,5 & 1,01 \\
Estrela Jaraguari & 4,25 & 6,5 & 0,78 \\
Indaiá & 1,6 & 2,5 & 0,79 \\
Santo Antônio & 5,5 & 7,75 & 0,71 \\
Piuva & 6,00 & 11,00 & 0,93 \\
Sete de Setembro & 8,78 & 10,00 & 0,58 \\
Barra Nova & 7,25 & 14,75 & 1,03 \\
Lagoa Azul & 3,2 & 18,25 & 2,89 \\
Ranildo da Silva & 7,43 & 11,25 & 0,77 \\
Eldorado (441) & 4,75 & 6,5 & 0,69 \\
Tejim & 15,58 & 30,5 & 0,99 \\
Eldorado II & 5,25 & 8,75 & 0,84 \\
Angélica & 8,5 & 22,25 & 1,33 \\
Volta Redonda Cut & 2,75 & 4,5 & 0,83 \\
Volta Redonda Fat & 5,38 & 17,75 & 1,67 \\
Santa Luzia & 5,25 & 6,75 & 0,65 \\
São João & 5,00 & 11,5 & 1,17 \\
Sucesso & 2,93 & 6,75 & 1,17 \\
\hline \multicolumn{2}{l}{ Produtividade Geral Média } & \\
\hline
\end{tabular}

Fonte: Autores.

Na Tabela 5 estão as áreas de produção em hectares, os números de cabeças de suínos (em ua) e as produtividades (em ua ha-1), das produções dos 23 assentamentos de MS que tinham criações de suínos em 2015. 
Tabela 5. Áreas de produção, número de cabeças de suínos (UA), produtividades e produtividade média de suínos nos assentamentos selecionados de MS, em 2015.

\begin{tabular}{|c|c|c|c|}
\hline Assentamento & $\begin{array}{l}\text { Área } \\
\text { (ha) }\end{array}$ & $\begin{array}{l}\text { Quantidade } \\
\text { (ua) }\end{array}$ & $\begin{array}{l}\text { Produtividade } \\
\text { Média (ua ha-1) }\end{array}$ \\
\hline Alambari & 0,95 & 13,75 & 14,47 \\
\hline Altemir Tortelli & 1,38 & 10,25 & 7,43 \\
\hline Corguinho & 0,50 & 0,50 & 1,00 \\
\hline Liberdade Camponesa & 0,25 & 0,50 & 2,00 \\
\hline Torre de Pedra & 0,75 & 5,25 & 7,00 \\
\hline Rancho Alegre & 0,13 & 0,75 & 6,00 \\
\hline Estrela Campo Grande & 2,49 & 5,00 & 2,01 \\
\hline Estrela Jaraguari & 0,25 & 3,00 & 20,00 \\
\hline Piuva & 0,38 & 1,25 & 3,33 \\
\hline Sete de Setembro & 0,13 & 1,00 & 8,00 \\
\hline Barra Nova & 0,35 & 4,75 & 13,77 \\
\hline Lagoa Azul & 0,10 & 1,75 & 17,50 \\
\hline Nazareth & 0,75 & 3,50 & 4,67 \\
\hline Ranildo da Silva & 0,25 & 2,00 & 8,00 \\
\hline Eldorado (441) & 0,38 & 1,50 & 4,00 \\
\hline Tejim & 0,24 & 0,25 & 1,04 \\
\hline Eldorado II & 0,30 & 1,50 & 5,00 \\
\hline Angélica & 0,55 & 2,00 & 3,64 \\
\hline Volta Redonda Cut & 0,50 & 1,00 & 2,00 \\
\hline Volta Redonda Fat & 0,50 & 5,25 & 10,50 \\
\hline Santa Luzia & 0,25 & 3,00 & 12,00 \\
\hline São João & 0,23 & 0,25 & 1,11 \\
\hline Sucesso & 0,25 & 3,00 & 20,00 \\
\hline
\end{tabular}

Produtividade Geral Média $\mathbf{7 , 5 9}$

Fonte: Autores.

Na tabela 6 estão anotadas as áreas de produção em hectares, os números de cabeças de aves (em ua) e as produtividades (em ua ha-1), das produções dos 30 assentamentos de MS que tinham criações de aves em 2015. 
Tabela 6. Áreas de produção, número de cabeças de aves (galinha e frangos), produtividades e produtividade média de aves nos assentamentos selecionados de MS, em 2015.

\begin{tabular}{|c|c|c|c|}
\hline Assentamento & $\begin{array}{l}\text { Ára } \\
\text { (ha) }\end{array}$ & $\begin{array}{l}\text { Quantidade } \\
\text { (ua) }\end{array}$ & $\begin{array}{l}\text { Produtividade Média } \\
\left(\text { ua ha }^{-1}\right)\end{array}$ \\
\hline Alambari & 0,85 & 68,75 & 80,88 \\
\hline Altemir Tortelli & 1,98 & 192,00 & 96,97 \\
\hline Corguinho & 6,88 & 19,25 & 2,80 \\
\hline Liberdade Camponesa & 3,10 & 10,75 & 3,47 \\
\hline Torre de Pedra & 3,25 & 13,25 & 4,08 \\
\hline Rancho Alegre & 3,13 & 20,00 & 6,39 \\
\hline Santa Mônica & 1,75 & 7,50 & 4,29 \\
\hline Estrela Campo Grande & 2,05 & 28,00 & 13,66 \\
\hline Estrela Jaraguari & 2,78 & 75,00 & 26,98 \\
\hline Indaiá & 1,84 & 15,00 & 8,15 \\
\hline Santo Antônio & 0,18 & 8,50 & 47,22 \\
\hline Piuva & 0,55 & 33,75 & 61,36 \\
\hline Sete de Setembro & 3,13 & 9,00 & 2,88 \\
\hline Barra Nova & 0,91 & 52,50 & 57,69 \\
\hline Lagoa Azul & 0,50 & 42,50 & 85,00 \\
\hline Nazareth & 6,14 & 29,25 & 4,76 \\
\hline Ranildo da Silva & 0,75 & 36,25 & 48,33 \\
\hline Eldorado (441) & 2,63 & 57,50 & 21,86 \\
\hline Tejim & 1,75 & 21,25 & 12,14 \\
\hline Eldorado II & 0,70 & 23,00 & 32,86 \\
\hline Angélica & 1,38 & 20,50 & 14,86 \\
\hline Volta Redonda Cut & 3,45 & 58,25 & 16,88 \\
\hline Volta Redonda Fat & 1,90 & 18,75 & 9,87 \\
\hline Santa Luzia & 3,43 & 35,75 & 10,42 \\
\hline São João & 1,13 & 34,00 & 30,09 \\
\hline Sucesso & 0,55 & 42,25 & 76,82 \\
\hline
\end{tabular}

Fonte: Autores.

De posse dos dados sobre as produtividades dos assentamentos relativas às produções agrícola e pecuária, dos níveis de produtividades ideais fornecidas por especialistas e, utilizando-se a ferramenta fuzzy do software Matlab, pode-se determinar qual o assentamento é produtivo, ou ainda, se o conjunto dos assentamentos de MS é produtivo. O acesso à ferramenta fuzzy é realizada digitando, após o prompt > o comando (>>fuzzy) na janela de comandos do MatLab (Matlab, 2019; Amendola et al., 2005). Uma janela denominada Fuzzy Logic Designer Unilled será aberta, conforme a Figura 1. 
Figura 1. Criação das variáveis de entradas e saída e as inferências da produção agrícola nos assentamentos de MS em 2015, da ferramenta fuzzy do software Matlab.

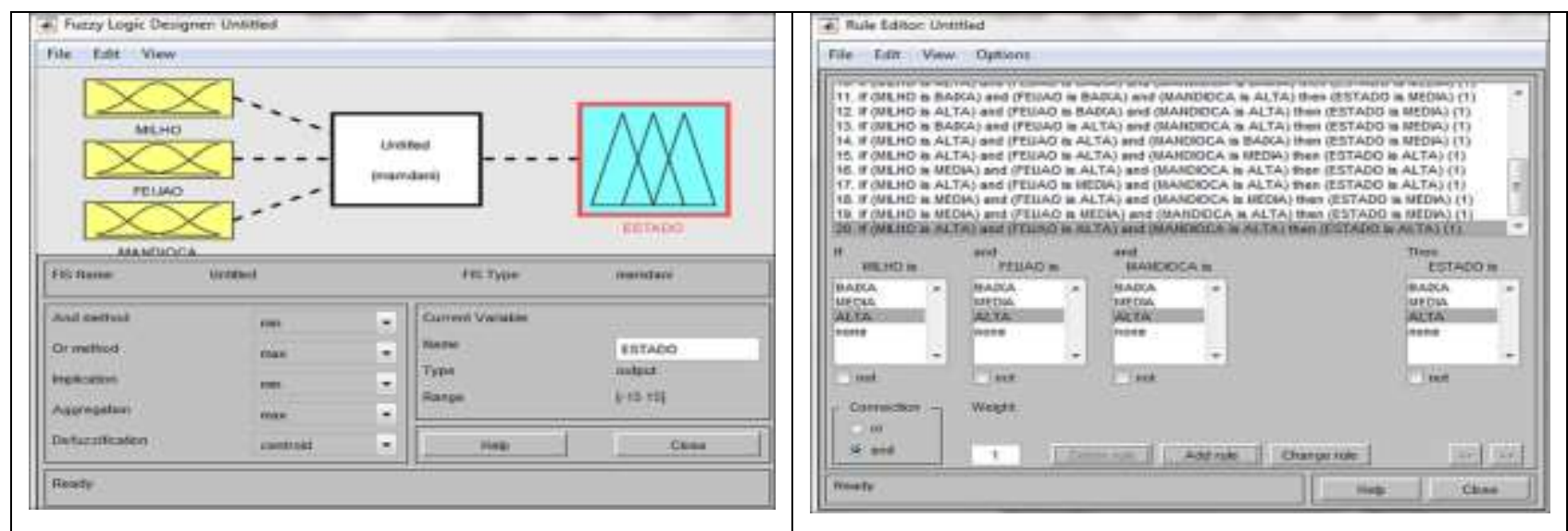

Fonte: Autores.

Conforme a Figura 1 as variáveis de entrada e saída foram criadas com três níveis de produtividade (Baixa, Média e Alta), para que seja testada na estrutura condicional proposta pela lógica fuzzy. Observe, ainda, na Figura 1 que as funções de pertinências das variáveis de entrada milho, feijão e mandioca são todas triangulares, bem como, a de saída (Estado), também é triangular. A Figura 2 apresenta um cenário resultante da seleção de valores das variáveis de entrada e o associado valor da variável de saída.

Figura 2. Um cenário resultante da seleção de valores das variáveis de entrada e o associado valor da variável de saída da produção agrícola dos Assentamentos de MS, em 2015.

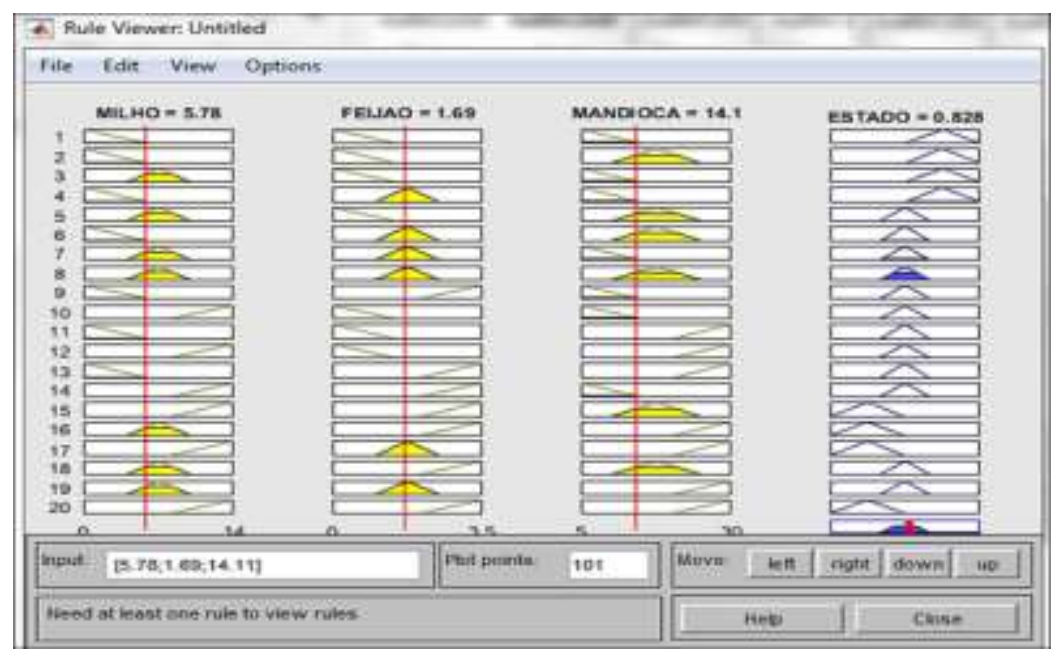

Fonte: Autores.

Foram atribuídos os valores das variáveis de entrada 1, 2 e 3, para as produções agrícolas (em t ha-1) de milho 5,78, feijão 1,69 e mandioca 14,11. Como saída, o sistema fuzzy gerou o valor 0,828 , cuja interpretação é a que permite o suporte à decisão (Quadro 3), de que é média a produtividade agrícola relativa aos Assentamentos de MS. A Figura 3 apresenta a criação das variáveis de entradas e saída da produção da pecuária na ferramenta fuzzy do software Matlab. 
Figura 3. Criação das variáveis de entradas e saída e as inferências da produção pecuária nos assentamentos de MS em 2015, da ferramenta fuzzy do software Matlab.

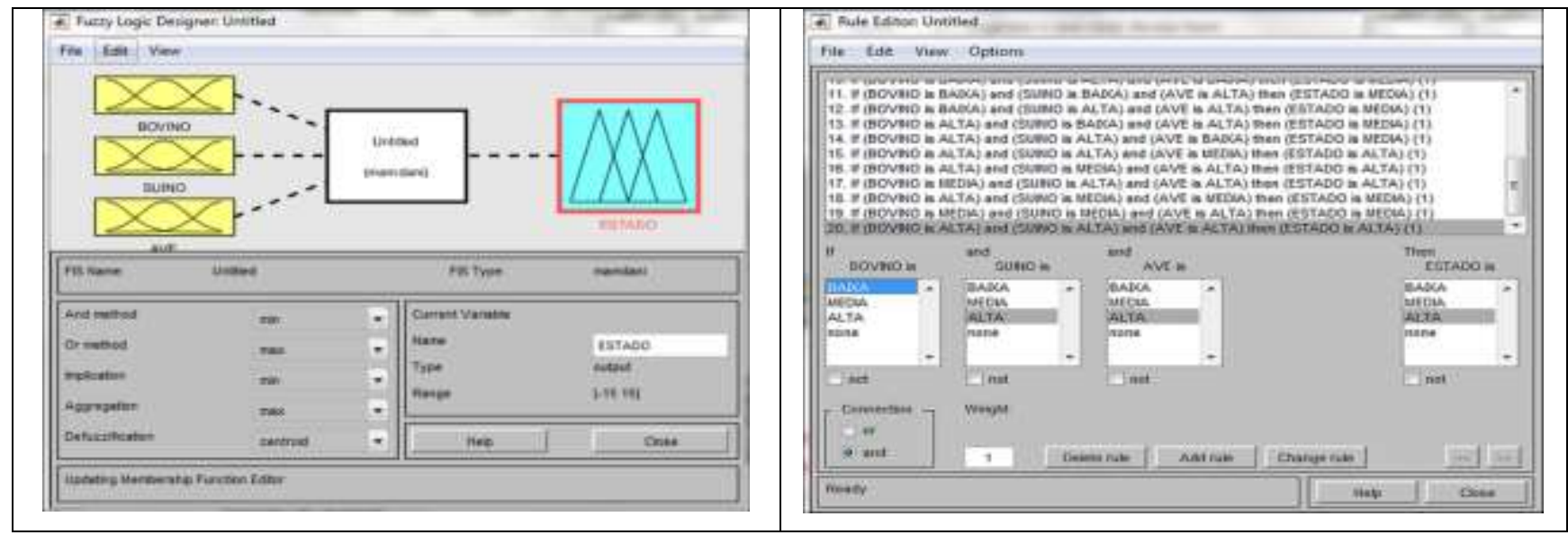

Fonte: Autores.

Observa-se, na Figura 3 que as funções de pertinências das variáveis de entrada (input) (bovino, suíno e aves), são todas triangulares, bem como, a de saída (output) (Estado), também é triangular. O próximo passo é entrar com os valores das variáveis de entrada no software para obter a saída desejada. Na Figura 4 foram atribuídos os valores das três variáveis de entrada para as produções da pecuária (em ua ha-1): bovina 1,5, suína 8,5 e aves 35. Como saída, o sistema fuzzy gerou o valor 0, cuja interpretação é a que permite o suporte à decisão, pelo Quadro 3, de que é média a produtividade da pecuária relativa aos Assentamentos de MS.

Figura 4. Um cenário resultante da seleção de valores das variáveis de entrada e o associado valor da variável de saída da produção da pecuária nos assentamentos de MS, em 2015.

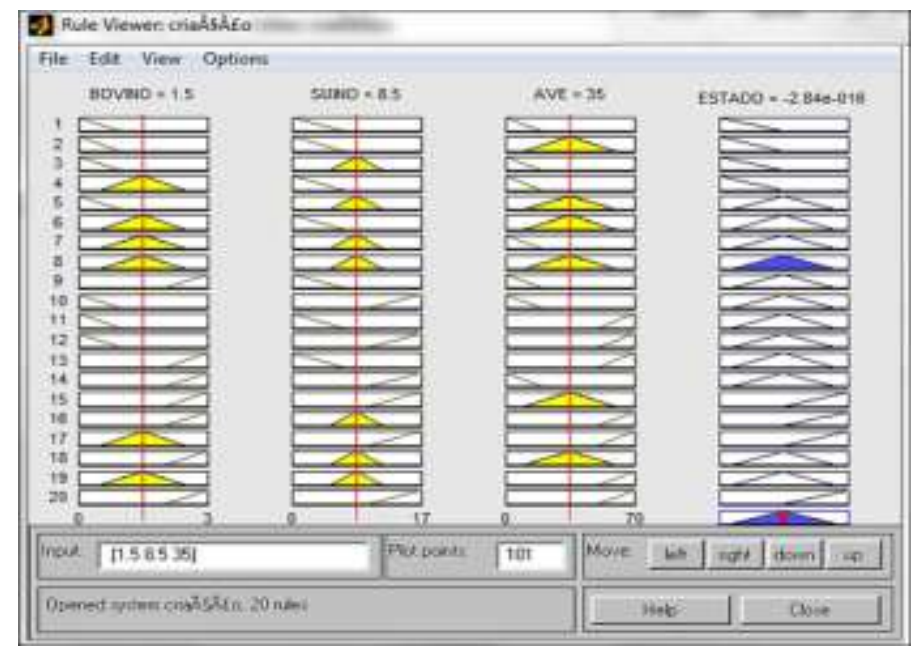

Fonte: Autores.

Como as produtividades tanto agrícola quanto na pecuária foram médias, conclui-se que o conjunto de Assentamentos de MS tem produtividade geral média. 


\section{Considerações Finais}

Verificou-se que alguns assentamentos têm altas produtividades na agricultura e na pecuária, como também, alguns assentamentos estão abaixo da produtividade mínima esperada. É possível fornecer aos Órgãos responsáveis pelos assentamentos em MS as informações necessárias para tornar produtivos aqueles assentamentos de baixas produtividades.

O resultado da simulação proposta neste trabalho pode ser considerado satisfatória, visto que a saída obtida com a aplicação da lógica fuzzy, para as estimativas de produtividades dos assentamentos rurais de MS, ficou constatado que esses assentamentos estão produzindo de forma mediana tanto no setor agrícola quanto na pecuária. A capacidade da lógica fuzzy em descrever ou classificar detalhes de forma gradual, permite uma aproximação muito maior da realidade que é marcada por ser um sistema complexo de muitas variáveis e valores ambíguos e inexatos.

Mediante o contexto e resultados apresentados, é possível e necessário a ampliação e discussão deste tema com novos estudos a fim de se esgotar ao máximo as possibilidades de uso da lógica fuzzy.

\section{Referências}

Amendola, M., Souza, A. L. de, \& Barros, L. C. (2005). Manual do uso da teoria dos conjuntos Fuzzy no MATLAB 6.5. FEAGRI \& IMECC. UNICAMP. http://www.logicafuzzy.com.br/wpcontent/uploads/2012/12/manual_fuzzy_matlab.pdf

Castelao, R. A., Souza, C. C., Frainer, D. M., \& Carneiro Junior, J. B. A. (2020). Crescimento econômico e indução de alteração ambiental no Mato Grosso do Sul entre 1991 e 2010. Revista Ibero-Americana de Ciências Ambientais, 11(4), 331-344.

CONAB. Companhia Nacional de Abastecimento (2007). Safra 2007. Brasília, 2007. https://www.conab.gov.br/OlalaCMS/arquivos

Coutinho, M., Bitencourt, M., Figueiredo-Neto, L., \& Figueiredo, L. A. (2019) Contribuição das atividades de base agropecuária na geração de emprego nos municípios de Mato Grosso do Sul (Brasil). Revista Latinoamericana de Estudios Urbano-Regionales, 45(135), $223-244$.

Cracolici, M. F., \& Cuffaro, M. (2010). The Measurement of Economic, Social and Environmental Performance of Countries: A Novel Approach. Social Indicators Research, 10(1), 339-356.

Bungenstab, D. J. (2012) Agronegócio com sustentabilidade: a eficiência das cadeias produtivas do agronegócio em Mato Grosso do Sul. Brasília, DF: Embrapa.

Fagundes, M. B. B., Dias, D. T., Figueiredo, A. M. R., \& Pitaluga, C. M. (2016) Interligações setoriais e composição do produto, demanda e renda da agropecuária de Mato Grosso do Sul: uma aplicação da matriz insumo-produto. Contextus Revista Contemporânea de Economia e Gestão, 14(1), 58-82.

IBGE. Instituto Brasileiro de Geografia e Estatística (2015). Produção Agrícola Municipal - PAM. Rio de Janeiro. https://www.ibge.gov.br/estatisticas/economicas/.

IBGE. Instituto Brasileiro de Geografia e Estatística (2015). Instituto Brasileiro de Geografia e Estatística. Contas Nacionais Brasil 2005:2009. Tabelas de Recursos e Usos.

IBGE. Instituto Brasileiro de Geografia e Estatística (2020). Instituto Brasileiro de Geografia e Estatística. Contas Regionais do Brasil.

Koche, J. C. (2011) Fundamentos de metodologia científica: teoria da ciência e iniciação à pesquisa. Editora Vozes.

Lima, A. C., Assis, J., Sayanda, D., Sabino, J., \& Oliveira, R. F. (2014). Impact of ecotourism on the fish fauna of Bonito region (Mato Grosso do Sul State, Brazil): ecological, behavioural and physiological measures. Neotropical Ichthyol, 12(1), 133-143.

MATLAB (2019). Fuzzy Logic Toolbox for use with MatLab - User's Guide. The Mathworks Inc.

Oliveira, R. D., Souza, C. C., \& Mercante, M. A. (2017). Análise e diagnóstico da sustentabilidade do assentamento rural Eldorado II, no município de Sidrolândia (MS). Informe GEPEC, 21(1), 149-168.

Ramos, F. S., Souza, C. C., Reis Neto, J. F., \& Frainer, D. M. (2015). Practical tool in classification of animals for slaughter by Fuzzy logic. African Journal of Agricultural Research, 10(7), 604-612. 